\title{
Sensorineural hearing loss and prematurity
}

\author{
Elaine S Marlow, Linda P Hunt, Neil Marlow
}

\begin{abstract}
Objective-To elucidate clinical antecedents of sensorineural hearing loss (SNHL) in very preterm infants.

Design-Case-control study.

Subjects-Fifteen children < 33 weeks' gestation with significant SNHL born between 1 January 1990 and 31 December 1994, detected within 9 months of birth, and 30 matched control children.

Methodology-Perinatal variables in the two groups were compared using nonparametric tests and conditional logistic regression (EGRET).

Results-Median birth weight for the index group was $960 \mathrm{~g}$ (range 600-2914 g) compared with $1026 \mathrm{~g}$ (range 410-2814 g) for controls. Children with SNHL had longer periods of intubation, ventilation, oxygen treatment, and acidosis, and more frequent treatment with dopamine or frusemide. Neither peak nor trough aminoglycoside levels, nor duration of jaundice or level of bilirubin varied between groups. However, SNHL was more likely if peak bilirubin levels coexisted with netilmicin use (odds ratio (95\% confidence interval) 14.2 (1.8 to 113.6$)$ ) or if acidosis occurred when bilirubin levels were over $200 \mu \mathrm{mol} / 1$ (OR 8.0 (0.9 to 71.6). Frusemide use in the face of high serum creatinine levels (OR $8.9(1.1$ to 74.5$)$ ) or netilmicin treatment (OR 5.0 (0.99) to 24.8)) was also associated with SNHL. At 12 months of age, seven of 15 children with SNHL had evidence of cerebral palsy compared with two of 30 controls (OR 12.3 (2.1 to 71)).
\end{abstract}

Conclusions-Preterm children with SNHL required more intensive care in the perinatal period and developed more neurological complications than controls. Among very preterm babies, the coexistence of risk factors for hearing loss may be more important than the individual factors themselves.

(Arch Dis Child Fetal Neonatal Ed 2000;82:F141-F144)

Hearing Assessment Centre, University of Bristol, Bristol, UK E S Marlow

Division of Child Health, University of Bristol

L P Hunt

N Marlow

Correspondence to: Professor E S Marlow, Department of Child Health, Level E East Block, Queens Medical Centre, Nottingham NG7 2UH, UK

Accepted 13 August 1999
Keywords: very preterm infants; hearing; sensorineural hearing loss; bilirubin; creatinine; aminoglycosides

Sensorineural hearing loss (SNHL) is found in a small proportion of very preterm survivors, but prematurity is a commonly quoted risk factor for acquired hearing loss. In various very low birthweight or preterm populations, the prevalence of high frequency hearing loss among survivors ranges from 0 to $4 \%,{ }^{1-3}$ about 10 times the incidence in unselected populations.
Various aetiological mechanisms have been suggested, including bilirubin and drug toxicity, hypoxic brainstem injury, haemorrhage into the inner ear, acoustic trauma, and cytomegalovirus infection. The causes of SNHL in very preterm infants may differ from those in more mature children as, in parallel with other neurological structures, the period between 20 and 33 weeks' gestation is one of rapid fetal audiological development. Among very preterm deaths, labyrinthine pathology may be found in a considerable number of cases. ${ }^{4}$

Previous studies have attempted to define antecedents of and associations with SNHL, but positive associations have not generally been confirmed, and methodological differences in populations and definitions of hearing loss make conclusions difficult. As prospective study is difficult in view of the relatively low prevalence, we undertook a case-control study of children born before 33 weeks' gestation in the Bristol area between 1990 and 1994 in order to determine which factors or combination of factors were associated with subsequently identified SNHL.

\section{Methods}

POPULATION

All children with SNHL of $50 \mathrm{dBnHL}$ (normal hearing loss) or greater on auditory brainstem response testing, born between 1 January 1990 and 31 December 1994 to families resident in the greater Bristol area, were identified from records of the Hearing Assessment Centre of the Royal Hospital for Sick Children, Bristol. Cases were included if the hearing loss had been identified within three months of discharge home. Of the 27 children of less than 33 weeks' gestation who met these criteria, eight were later found to have essentially a conductive hearing loss, two had abnormalities of the head and neck indicating a possible congenital cause, one had had neonatal bacterial meningitis, and the case records of one case could not be identified. Fifteen children (11 girls, four boys, seven twins) therefore comprised the index group. Later behavioural testing (distraction test, visual reinforcement, or pure tone audiometry, as appropriate to the child's development) confirmed appreciable hearing loss in all 15 index children. Fourteen were fitted with and used hearing aids (the parents of the remaining child with high frequency loss did not wish to use aids). Two children (both girls) had bilateral high frequency SNHL (moderate, loss of 41-70 dBHL (hearing loss) at 4000 $\mathrm{Hz}$ ), one of whom had cerebral palsy; five girls had bilateral moderate SNHL (averaged over $500-4000 \mathrm{~Hz}$ ), four with cerebral palsy; three children (two boys) had bilateral severe SNHL (71-95 dBHL), one boy with cerebral palsy; 
five children (two boys) had bilateral profound SNHL (> $95 \mathrm{dBHL}$ ), one girl with cerebral palsy. Cerebral palsy was predominantly of the spastic type with significant disability.

Two comparison children were chosen for each index case as survivors matched on the hospital providing neonatal intensive care, sex, and gestational age. These children were identified as the next and preceding matching children in the admission books of St Michael's and Southmead Hospitals, Bristol. Although seven index cases were twins, we decided to match these with singletons as matching would otherwise have been difficult and we are not aware of any suggestion in the available literature that twins are at greater risk of SNHL. All control children had passed local hearing screening tests and had normal hearing across the speech frequency range at follow up. Median gestational age for both groups was 28 (range 26-31) weeks.

COLLECTION OF DATA

The clinical and nursing records of the two groups were scrutinised, and data were abstracted on to a predefined proforma. Quantification of two aspects of the neonatal course, the time course of hypoxia and the rate of increase in bilirubin, was not possible because of the methods of recording and variability of sampling sites and times. Two groups of data were identified, conventional descriptors of course (condition at birth, CRIB score (CRIB
1990), duration of ventilation, oxygen treatment, etc) and daily occurrence of risk factors (bilirubin, ototoxic drugs, acidosis). The cooccurrence of risk factors in the same 24 hour period was then identified.

Data were then entered on to the computer and analysed with non-parametric tests using SPSS for Windows (version 7.1) and conditional logistic regression using the EGRET software package.

\section{Results}

Table 1 shows the clinical variables. Birth weight, condition at birth, and CRIB scores did not differ significantly between groups. However, children who developed SNHL received intubation, ventilation, and oxygen treatment for longer, and had longer periods of acidosis, defined as the length of time in hours with $\mathrm{pH}$ $<7.2$ or with base deficit $>9.0 \mathrm{mmol}$. They were more likely to have received dopamine $(p=0.040)$ or frusemide $(p=0.010)$. Commensurate with this, 10 index children had cerebral ultrasound abnormalities compared with only four controls $(\mathrm{p}<0.001)$. Bilirubin levels were measured in 14 index/28 control children, and the distribution of peak levels measured was similar in the two groups. The proportion of children with bilirubin levels $>200 \mu \mathrm{mol} / 1$ or with a bilirubin level over an arbitrary "exchange level" in $\mu \mathrm{mol} / 1$ of 10 times the gestational age in weeks was also similar. Thirteen of 15 index children and 24 of 30

Table 1 Comparison between single perinatal variables

\begin{tabular}{|c|c|c|c|c|c|}
\hline & \multicolumn{2}{|c|}{ SNHL group $(n=15)$} & \multicolumn{2}{|c|}{ Comparison group $(n=30)$} & \multirow[b]{2}{*}{ Likelihood ratio test } \\
\hline & Median & Range & Median & Range & \\
\hline Birth weight (g) & 960 & $600-2914$ & 1026 & $410-2814$ & $\mathrm{p}=0.667$ \\
\hline \multicolumn{6}{|l|}{ Apgar score } \\
\hline At $1 \mathrm{~min}$ & 5 & $1-8$ & 5.5 & $1-9$ & $p=0.216$ \\
\hline At $5 \mathrm{~min}$ & 8 & $2-9$ & 8.5 & $3-10$ & $\mathrm{p}=0.156$ \\
\hline CRIB score & 4 & $1-9$ & 2 & $0-12$ & $\mathrm{p}=0.512$ \\
\hline Maximum serum bilirubin $(\mu \mathrm{mol} / \mathrm{l}) \dagger$ & $\begin{array}{l}\mathrm{n}=14 \\
239\end{array}$ & $148-473$ & $\begin{array}{l}\mathrm{n}=28 \\
232.5\end{array}$ & $130-366$ & $\begin{array}{l}\text { (14 matched sets) } \\
\mathrm{p}=0.174\end{array}$ \\
\hline \multicolumn{6}{|l|}{ Duration of } \\
\hline Intubation (days) & 14 & $4-41$ & 2 & $0-48$ & $p=0.011^{\star}$ \\
\hline Respiratory support (days) $\ddagger$ & 34 & $9-93$ & 5.5 & $0-67$ & $\mathrm{p}=0.002^{\star}$ \\
\hline Added $\mathrm{O}_{2}$ (days) & 57 & $2-140$ & 9.5 & $0-120$ & $\mathrm{p}=0.004^{\star}$ \\
\hline $\mathrm{pH}<7.2(\mathrm{~h})$ & 12 & $0-85$ & 0.5 & $0-28.5$ & $\mathrm{p}=0.001^{\star}$ \\
\hline Base excess $<-9.0(\mathrm{~h})$ & 9 & $0-57$ & 0 & $0-58$ & $\mathrm{p}=0.034^{\star}$ \\
\hline Netilmicin & $\mathrm{n}=13$ & & $\mathrm{n}=24$ & & (13 matched sets) \\
\hline Peak (mg/l) & 6.6 & $5.4-10$ & 6.4 & $2.9-9.4$ & $\begin{array}{l}\mathrm{p}=0.357 \\
(11 \text { matched sets })\end{array}$ \\
\hline Trough (mg/l) & 2.5 & $1.2-4.9$ & 2.3 & $0.6-5.2$ & $\mathrm{p}=0.252$ \\
\hline Vancomycin & $\mathrm{n}=4$ & & $\mathrm{n}=4$ & & (1 matched sets) \\
\hline Peak (mg/1) & 34.3 & $19.3-43.1$ & 25.7 & $17.8-46.8$ & I \\
\hline \multirow[t]{2}{*}{ Trough (mg/l) } & 13.85 & $3.5-18$ & 6.2 & $5.2-20.9$ & ๆ \\
\hline & $n$ & $\%$ & $n$ & $\%$ & Odds ratio $(95 \%$ CI) \\
\hline Received ventilation & 13 & 87 & 22 & 73 & $2.3(0.4-12.2)$ \\
\hline Positive blood culture & 11 & 73 & 14 & 47 & $7.0(0.8-60.2)^{\star}$ \\
\hline Bilirubin $>200 \mu \mathrm{mol} / 1 \S$ & $12 / 14$ & 86 & $22 / 28$ & 79 & $2.0(0.4-10.6)$ \\
\hline Bilirubin $>\mathrm{GA} \times 10 \Omega$ & $2 / 14$ & 14 & $4 / 28$ & 14 & $1.0(0.2-5.5)$ \\
\hline Creatinine $>60 \mathrm{mmol} / 1$ & $12 / 14$ & 86 & 18 & 60 & ब \\
\hline \multicolumn{6}{|l|}{ Treated with } \\
\hline Dopamine & 5 & 33 & 2 & 7 & $5.0(1.0-25.8)^{\star}$ \\
\hline Frusemide & 13 & 87 & 16 & 53 & $9.7(1.2-81.8)^{\star}$ \\
\hline Indomethacin & 4 & 27 & 8 & 27 & $1.0(0.2-4.3)$ \\
\hline Netilmicin & 15 & 100 & 26 & 87 & ब \\
\hline Vancomycin & 6 & 40 & 5 & 17 & $4.0(0.8-20.9)$ \\
\hline Abnormal cerebral ultrasound scan & 10 & 67 & 4 & 13 & $15.6(2.0-124.3)^{\star}$ \\
\hline
\end{tabular}

${ }^{\star} \mathrm{p}<0.05$ on likelihood ratio test, including positive blood culture, as $95 \%$ CI values are approximate.

†No bilirubin results were available for one index and two control children (14 matched sets).

$\neq$ Includes continuous positive airways pressure.

\Arbitrary exchange level $=$ bilirubin concentration $>$ gestational age $\times 10$.

ๆConvergence not possible, neither significant using Fisher's exact test (does not take account of matching). 
Table 2 Results of conditional logistic regression analysis (CLR)

\begin{tabular}{|c|c|c|c|c|}
\hline & $\begin{array}{l}\text { SNHL group } \\
(n=15)^{\star} n(\%)\end{array}$ & $\begin{array}{l}\text { Comparison group } \\
(n=30)^{\star} n(\%)\end{array}$ & Matched setst & $\begin{array}{l}\text { Odds ratio (CLR) } \\
\text { ( } 95 \% \text { CI) }\end{array}$ \\
\hline Bilirubin $>200+$ acidosis & $4 / 13(30.8)$ & $1 / 29(3.5)$ & 13 & $8.0(0.9-71.6)$ \\
\hline Bilirubin $>200+$ sepsis & $2 / 14(14.3)$ & $3 / 29(10.3)$ & 14 & $1.2(0.2-7.0)$ \\
\hline Bilirubin $>200+$ netilmicin & $10 / 14(71.4)$ & $5 / 29(17.2)$ & 14 & $14.2(1.8-114)$ \\
\hline Bilirubin $>200+$ vancomycin & $1 / 14(6.7)$ & $0(0)$ & & $\ddagger$ \\
\hline Bilirubin $>200+$ frusemide & $4 / 14(26.7)$ & $3(10)$ & 14 & $3.3(0.6-18.9)$ \\
\hline Peak bilirubin + acidosis & $2 / 14(14.3)$ & $1 / 28(3.6)$ & 14 & $2.6(0.2-29.1)$ \\
\hline Peak bilirubin + sepsis & $4 / 14(28.6)$ & $2 / 28(7.1)$ & 14 & $3.6(0.7-19.9)$ \\
\hline Peak bilirubin + netilmicin & $13(86.6)$ & $4 / 28(14.3)$ & & $\ddagger$ \\
\hline Peak bilirubin + frusemide & $5(33.3)$ & $3 / 29(10.3)$ & 14 & $4.3(0.8-22.8)$ \\
\hline Creatinine $>60+$ netilmicin & $11 / 14(78.6)$ & $21(70)$ & 14 & $1.5(0.3-7.5)$ \\
\hline Creatinine $>60+$ vancomycin & $3 / 14(21.4)$ & $3(10)$ & 14 & $3.4(0.3-35.8)$ \\
\hline Creatinine $>60+$ frusemide & $9 / 14(64.3)$ & $8(26.7)$ & 14 & $8.9(1.1-74.5)$ \\
\hline Netilmicin + frusemide & $10(66.6)$ & $11(36.7)$ & 15 & $5.0(0.99-24.8)$ \\
\hline Vancomycin $\+$ frusemide & $4(26.6)$ & $4(13.3)$ & 15 & $2.7(0.5-15.9)$ \\
\hline
\end{tabular}

Data in bold are significant $(\mathrm{p}<0.05)$. Creatinine $(\mathrm{mmol} / \mathrm{l})=$ bilirubin $(\mu \mathrm{mol} / \mathrm{l})$.

$\star$ Unless otherwise stated.

tNumber of sets used for CLR analysis.

¥Convergence not possible.

$\S$ No patients in the SNHL group or the comparison group were administered vancomycin at the time of peak bilirubin levels.

SNHL, sensorineural hearing loss.

controls received netilmicin, and five in each group received vancomycin. Peak and trough levels were similarly distributed between groups (table 1). Renal function as indicated by serum creatinine levels was also similar. Positive blood cultures and frusemide usage were also more common in the SNHL group.

To evaluate the effect of combinations of risk factors, we examined the independent effects of bilirubin $>200 \mu \mathrm{mol} / 1$ or creatinine $>60$ $\mathrm{mmol} / \mathrm{l}$ when they occurred at the same time as other risk factors, namely acidosis, positive blood culture, aminoglycoside, and frusemide use. In addition, we assessed the effect of the presence of these factors on the day the bilirubin was highest (peak bilirubin) and on days when two potentially ototoxic drugs were administered together (netilmicin, vancomycin, frusemide). Using combinations of two risk factors (table 2), four combinations occurred more often in the SNHL group (bilirubin $>200 \mu \mathrm{mol} / 1+$ acidosis) $(\mathrm{p}=0.032)$, (bilirubin $>200 \mu \mathrm{mol} / 1+$ netilmicin) ( $p<0.001)$, (frusemide + netilmicin) $(\mathrm{p}<0.033)$, and (creatinine $>60 \mathrm{mmol}+$ frusemide) $(p<0.012)$. The incidence of other combinations did not differ significantly between groups.

At 12 months after the expected date of discharge, seven of the SNHL group (47\%) and two of the controls $(7 \%)$ had a clinical diagnosis of cerebral palsy (Fisher's exact test: $\mathrm{p}=0.022$ ).

\section{Discussion}

SNHL remains a distinct cause of disability in surviving very preterm children and is particularly associated with variables that indicate a severe respiratory course (duration of ventilation and oxygen treatment) despite there being no differences in birth weight, condition at birth, or initial disease severity. In terms of aetiology this fact is not helpful, as a longer respiratory illness may also be associated with greater exposure to risk factors, such as acidosis, antibiotic courses, and incubator or ventilator noise, and is also associated with neurological injury. Abramovich and colleagues ${ }^{5}$ found apnoeic spells more often in very low birthweight infants with SNHL than in unmatched low birthweight children. They developed the hypothesis that recurrent hypoxia was important in the genesis of acquired SNHL. Three studies have identified ventilation and oxygen treatment with increased risk of hearing loss, ${ }^{6-8}$ although one study also included children with conductive losses. ${ }^{8}$ The aetiological pathway has not, however, been defined. The introduction of high frequency ventilation was initially associated with fears that noise induced losses may be produced, but this appears not to be the case. ${ }^{9}$

The association of high bilirubin levels with SNHL has its origins in the association between bilirubin encephalopathy and subsequent deafness. In neonatal studies, bilirubin levels appear to cause delay in auditory brainstem response latencies, indicating changes in upper auditory pathways, which return to normal as bilirubin levels fall. ${ }^{10}{ }^{11}$ The relation between high bilirubin levels and

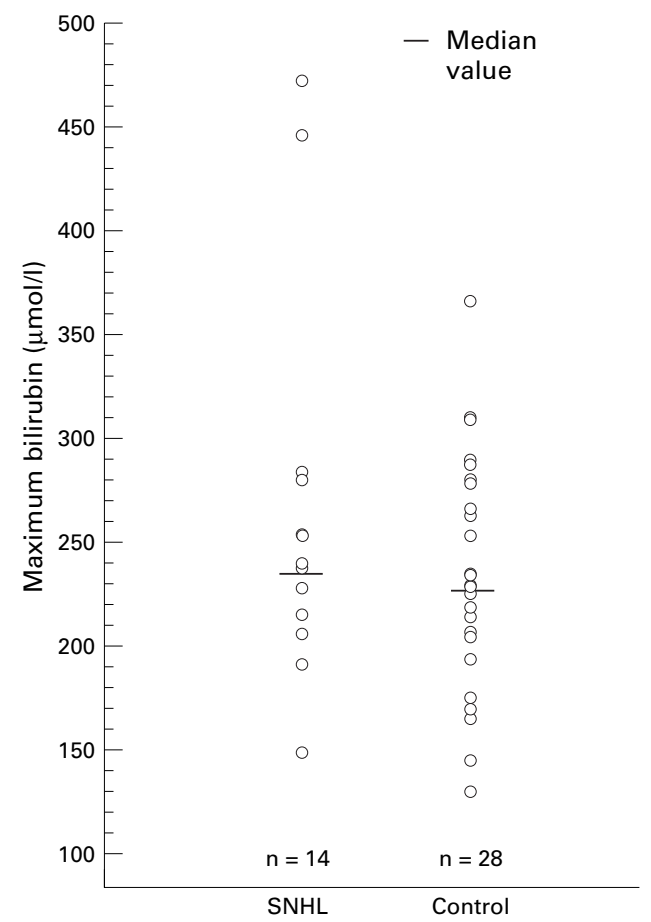

Figure 1 Maximum serum bilirubin levels in index cases (sensorineural hearing loss (SNHL)) and controls. 
SNHL has been explored in several studies, all with slightly different methodologies. In low birthweight populations, associations have been observed between a range of hearing impairments at 8 years and high bilirubin levels, ${ }^{7}$ and between the maximum bilirubin concentration and SNHL. ${ }^{12}$ De Vries and colleagues $^{13}$ reported an interaction between birth weight, bilirubin concentration, and SNHL in a group of children $<34$ weeks' gestation with serum bilirubin levels $>240 \mu \mathrm{mol} / 1$. In this population study, there was little evidence of a direct relation between bilirubin and SNHL (fig 1), no excess of children who triggered a $200 \mu \mathrm{mol} / 1$ threshold, or in those whose maximum bilirubin exceeded a notional exchange transfusion level equal to 10 times the gestational age in weeks. Sixteen children met the criteria of de Vries et al for inclusion, 10 of whom were controls. Thus nine of 15 index children had maximum serum bilirubin levels under $240 \mu \mathrm{mol} / 1$. It was difficult to plot area under the bilirubin curve or to determine the maximum rate of increase with any accuracy because of poor recording of the time of sampling, which may have shed extra light on a possible relation.

Given that it is likely that high bilirubin levels provide an increase in background risk of SNHL, it is logical to examine the data for situations where risk factors coincide. Previous studies have not examined the timing of risk factors with respect to bilirubin level. In this study, we identify increased risk of SNHL with high bilirubin levels in the presence of concomitant acidosis or netilmicin use. Furthermore, the use of frusemide in association with increased creatinine was associated with increased risk of SNHL. Each association is logical.

In the presence of acidosis, unconjugated bilirubin is displaced from albumin, increasing the free bilirubin concentration and hence the risk of neuronal damage. Only de Vries et $a l^{13}$ have previously noted such an association within their selected group with relatively high bilirubin levels.

The use of aminoglycosides has been studied previously, first by Yow et $a l^{14}$ in 1962, who showed that the risk of hearing loss was increased with cumulative kanamycin doses above $500 \mathrm{mg} / \mathrm{kg}$ with normal renal function, seeming to confirm adult and animal evidence for ototoxicity and interaction between aminoglycoside and other factors, such as acoustic trauma. Suggestions of aminoglycoside toxicity ${ }^{12}{ }^{13}$ have not been confirmed, and a prospective study comparing infants treated with kanamycin and gentamicin with unexposed controls failed to show any association despite a large trial group. ${ }^{15}$ The interaction identified in the present study suggests that the timing of antibiotic administration with respect to the bilirubin level may be important, and not identifiable from previous attempts at multivariate analysis.

In one previous study an association between neonatal frusemide use and SNHL has been suggested. ${ }^{16}$ The interaction between aminoglycosides and loop diuretics is well described in animal models. ${ }^{17-20}$ In the guinea pig model the simultaneous administration of two commonly used neonatal drugs, gentamicin and frusemide, produces a rapid and profound loss of cochlear function, through a specific pattern of cochlear injury, different from the well described changes of aminoglycoside toxicity. ${ }^{18}$ In our study, frusemide use in conjunction with netilmicin was associated with SNHL. The effect of frusemide was also shown in the presence of slightly raised serum creatinine levels.

SNHL remains a major disability among very preterm children. In almost half the children in our cohort, it was associated with other neurological morbidity. From our data it would appear that the evaluation of jaundice in such children in the presence of acidosis and aminoglycoside treatment should be cautious and that frusemide should be avoided if creatinine levels are high or there is concomitant use of aminoglycoside. Further evaluation of interaction between risk factors in the generation of neonatally acquired hearing loss is necessary, in particular evaluation of more precise time weighted measures would seem warranted.

1 Cooke RWI. Annual audit of three year outcome in very low birthweight infants. Arch Dis Child Fetal Neonatal Ed birthweight infant

2 Robertson C, Sauve RS, Christianson HE. Province based study of neurologic disability among survivors weighing 500 through to $1249 \mathrm{~g}$ at birth. Pediatrics 1994;93:636-40.

3 Victorian Infant Collaborative Study Group. Eight year outcome in infants with birth weights of 500-999 g: continuing regional study of 1979 and 1980 births. F Pediatr 1991;118:761-7.

4 Hoing R. Inner ear changes in fetal asphyxia. Laryngorhinotologie 1991;70:72-7.

5 Abramovich SJ, Gregory S, Slemick M, Stewart A. Hearing loss in very low birthweight infants treated with neonatal intensive care. Arch Dis Child 1979;56:421-6.

6 Veen S, Sassen ML, Schreuder AM, et al. Hearing loss in preterm and very low birth weight infantsat the age of 5 years in a naotional cohort. Int $\mathcal{F}$ Pediatr Otorhinolaryngol years in a naotional $1993 ; 26: 11-28$.

7 Doyle LW, Keri E, Kitchen WH, Ford GW, Rickards AL, Kelly EA. Audiologic assessment of extremely low birthweight infants: a preliminary report. Pediatrics 1992;90:744-9.

8 Tudehope D, Smyth V, Scott J, Rodgers Y. Audiological evaluation of very low birthweight infants. Fournal of Paediatrics and Child Health 1992;28:172-5.

9 Cox LC, Martin RJ, Carol WA, Hack M. Early ABRs in infants undergoing assisted ventilation. $\mathcal{F}$ Am Acad Audiol 1993;4:13-17.

10 Meredith R, Stephens D, Hogan S, Cartlidge PHT, Drayton $\mathrm{M}$. Screening for hearing loss in an at-risk neonatal popu-
lation using evoked oto-acoustic emmisions. Scand Audiol lation using evoked

11 Bhandari V, Narang A, Mann SB, Raghunathan M, Bhakoo ON. Brain stem electrical response audiometry in neonates with hyperbilirubinaemia. Indian $\mathcal{f}$ Paediatr 1993;60:409-13.

12 Bergman I, Hirsch RP, Fria TP, Shapiro SM, Holzman I, Painter MJ. Causes of hearing loss in the high risk premature infant. F Pediatr 1985;106:95-101.

13 de Vries L, Lary S, Dubowitz LMS. Relationship of serum bilirubin levels to ototoxicity and deafness in high risk low birthweight infants. Pediatrics 1985;76:351-4.

14 Yow MD, Tengg NE, Bangs J, et al. The ototoxic effects of kanamycin sulfate in infants and children. Pediatrics 1962;60:230.

15 Finitzo-Hieber T, McCracken GH, Roeser RJ, Allen DA, Chrane DF, Morrow J. Ototoxicity in neonates treated with gentamicin and kanamycin: results of a four year conwith gentamicin and kanamycin: results of a four year
trolled follow up study. Pediatrics 1979;63:443-9.

16 Brown DR, Watchk JF, Sabo D. Neonatal sensorineural hearing loss associated with furosemide: a case control study. Dev Med Child Neurol 1991;33:816-23.

17 Hayashida T, Hiel H, Dulon D, Erre JP, Guilhame A, Aran JM. Dynamic changes following combined treatment with gentamicin and ethacrynic acid with and without acoustic stimulation [abstract]. Acta Otolaryngol 1989;108:404-13.

18 Mulheran M, Harpur ES. The effect of gentamicin and furosemide given in combination on cochlear potentials in the guinea pig [abstract]. Br $\mathcal{F}$ Audiol 1998;32:47-56.

19 Russell NJ, Fox KE, Brummett RE. Ototxic effects of the interaction between kanamycin and ethacrynic acid interaction between kanamycin and ethact

20 West BA, Brummett RE, Himes DL. Interaction of kanamycin with ethacrynic acid: severe cochlear damage in guinea pigs [abstract]. Arch Otolaryngol 1973;98:32-7. 